\title{
() OPEN ACCESS \\ Impact of direct-access IBD physician delivered endoscopy on clinical outcomes: a pre-implementation and post-implementation study
}

\author{
Matthew Stammers (D) , 1,2 Sohail Rahmany (D) ,1,3 Louise Downey, ${ }^{1}$ \\ Florina Borca, ${ }^{2,4}$ Clare Harris, ${ }^{1,3}$ Richard Harris, ${ }^{1,3}$ Martin McDonnell, ${ }^{1,3}$ \\ Stephanie Sartain, ${ }^{1,3}$ Nicolas Coleman, ${ }^{1}$ Bernard Stacey, ${ }^{1}$ \\ Trevor R Smith (D) , ${ }^{1,3}$ Fraser Cummings (D) , ${ }^{1,3}$ Richard Felwick, ${ }^{1}$ \\ Markus Gwiggner ${ }^{1,5}$
}

- Additional supplemental material is published online only. To view, please visit the journal online (http://dx.doi. org/10.1136/flgastro-2021 102047).

For numbered affiliations see end of article.

\section{Correspondence to}

Dr Markus Gwiggner,

Department of Gastroenterology, University Hospital Southampton NHS Foundation Trust, Southampton, UK; markus. gwiggner@uhs.nhs.uk

MS and SR are joint first authors.

Received 5 November 2021 Accepted 8 February 2022

\section{A Check for updates}

(C) Author(s) (or their employer(s)) 2022. Re-use permitted under CC BY-NC. No commercial reuse. See rights and permissions. Published by BMJ.

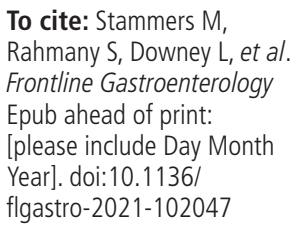

\begin{abstract}
Introduction Patients with suspected inflammatory bowel disease (IBD) referred from primary care often face diagnostic and treatment delays. This study aimed to compare a novel direct-access IBD endoscopy pathway with the traditional care model.

Method Single centre real-world study analysing primary care referrals with suspected IBD. Group A: patients triaged to direct-access IBD endoscopy. Group B: patients undergoing traditional outpatient appointments before the availability of direct-access IBD endoscopy. Demographics, fecal calprotectin (FCP), Creactive protein (CRP), disease activity score, endoscopy findings, treatment and follow-up were collected and statistically analysed. Ranked semantic analysis of IBD symptoms contained within referral letters was performed.

Results Referral letters did not differ significantly in Groups A and B. Demographic data, FCP and CRP values were similar. Referral to treatment time (RTT) at the time of IBD

\section{Significance of this study}

What is already known on this topic

- Inflammatory bowel disease (IBD) standards recommend that suspected IBD referrals are seen within 4 weeks.

- Nationally only $30 \%$ meet this target.

- Lower gastrointestinal endoscopy is the gold standard for the diagnosis of IBD.

What this study adds

- Key information to suspect a diagnosis of IBD is often contained in referral letters.

- Direct-access IBD physician-delivered endoscopy has a high yield of IBD diagnosis.

- Referral to treatment time fell by $86 \%$, introducing this novel pathway.

How might it impact on clinical practice in the foreseeable future

- Direct-access IBD endoscopy could be replicated elsewhere, facilitating adherence to national IBD benchmarking targets.
\end{abstract} endoscopy was reduced from 177 days (Group B) to 24 days (Group A) $(p<0.0001)$. Diagnostic yield of IBD was $35.6 \%$ (Group B) versus $62.0 \%$ (Group A) ( $p=0.0003$ ). $89.2 \%$ of patients underwent colonoscopy in Group B versus $46.4 \%$ in Group A. DNA rates were similar in both groups. The direct to IBD endoscopy pathway saved $100 \%$ of initial IBD consultant clinics with a 2.5-fold increase in IBD nurse-led follow-up.

Conclusion Our novel pathway resulted in an $86 \%$ reduction in RTT with associated increased diagnostic yield while saving $100 \%$ of initial IBD consultant outpatient appointments. Replication in other trusts may improve patient experience and accelerate time to diagnosis/treatment while optimising the use of healthcare resources.

\section{INTRODUCTION}

A survey by Crohn's and Colitis UK, using the inflammatory bowel disease (IBD) benchmarking tool, demonstrated that only $30 \%$ of primary care referrals with suspected IBD were seen by an IBD specialist within 4 weeks, failing the proposed IBD standard. ${ }^{1}{ }^{2}$ Recent UK evidence has revealed that fewer than $50 \%$ of patients subsequently diagnosed 
with IBD were seen in specialist secondary care within 18 months. ${ }^{3}$ NHS England has issued guidance on the transforming of elective gastroenterology services by encouraging clinicians to rethink established referral models to reduce waiting times, avoid unnecessary appointments while improving patient outcomes. ${ }^{4}$

The traditional care model for patients with suspected IBD referred from primary care consists of an outpatient appointment with subsequent endoscopic assessment. Endoscopic investigation with flexible sigmoidoscopy or colonoscopy performed by a specialist is the gold standard for clinical diagnosis of IBD, followed by subsequent histological confirmation. $^{5-7}$

We previously introduced direct-access IBD specialist endoscopy slots at University Hospital Southampton Foundation Trust (UHSFT) for patients with selected IBD contacting the IBD helpline. Changing the service delivery model for acute IBD flares by combining IBD physician-delivered endoscopy with proactive management decisions at the time resulted in a change in management for $84.9 \%$, while reducing outpatient appointments. $^{8}$

At UHSFT, we developed a novel electronic grading system to display relevant information about primary care referrals at the point of triage, enabling rapid decision-making. ${ }^{9}$ Referral letters usually contain relevant clinical information to suspect IBD. Therefore, for appropriately triaged patients with suspected IBD, a short consultation during direct-access IBDphysician delivered endoscopic assessment could make traditional first outpatient appointments redundant.

Combining the electronic grading system with direct-access IBD-physician delivered endoscopy for appropriate patients with suspected IBD could reduce the time to IBD diagnosis and treatment.

Natural language processing (NLP) has been proposed as a potential tool to retrieve clinically meaningful information from free-text documents in gastroenterology. ${ }^{10-12}$

The study aimed to evaluate the impact of introducing a direct-access IBD-physician delivered endoscopy pathway for new primary care referrals with suspected IBD.

\section{DESIGN}

\section{Study design and patients}

We conducted a single centre, pre-implementation and post-implementation study at UHSFT. We analysed the records of all patients referred with suspected IBD from primary care between 1 October 2016 and 31 March 2021.

Direct-access, IBD-physician delivered endoscopy pathway The direct-access IBD pathway (online supplemental figure 1) was introduced at the trust on 1 January 2019 for all suitable new primary care referrals with suspected IBD (patients with suspected IBD and new to service patients with symptomatic IBD). All referrals were graded by a consultant gastroenterologist according to an internally developed grading consensus guideline. If IBD was suspected, appropriate patients were booked directly onto specialised IBD physician-delivered endoscopy lists, sent an explanatory invitation letter (online supplemental figure 2) and contacted by telephone prior to appointment with an opportunity to opt out.

Four dedicated IBD consultant physicians performed all procedures in the study. Each patient underwent a short clinical assessment before endoscopic evaluation. An additional 1 point was added to each colonoscopy and 0.5 points to each flexible sigmoidoscopy. If diagnosed with IBD, treatment was initiated and further as appropriate and a specialist IBD nurse reviewed patients post procedure to provide relevant support and information.

\section{Study cohorts}

The direct to endoscopy group (Group A) consisted of consecutive patients triaged direct to IBD endoscopy between 1 January 2019 and 31 March 2021.

The control group (Group B) consisted of all patients with suspected IBD referred between 1 October 2016 and 31 December 2018 seen first in an IBD clinic with a subsequent lower gastrointestinal endoscopic investigation.

Group A received written communication explaining the rationale for the direct-access test approach, while Group B received a standard outpatient clinical invitation.

\section{Outcomes}

The primary outcome measure was referral to treatment time (RTT). ${ }^{13}$ RTT was defined as the time in days from referral receipt to date of endoscopic diagnosis and treatment.

Secondary outcome measures included diagnostic yield of IBD, type of endoscopy performed, IBD treatment and follow-up. Post- implementation patient feedback on the pathway has also been collected.

\section{Data collection and handling}

Baseline data were collected and pseudonymised from structured electronic health records as per (table 1).

To semantically compare the referral letter contents between the groups, we retrospectively deployed an NLP algorithm (MedCAT V.0.4.2). ${ }^{1014} 15$ These results were confirmed by two clinicians manually validating 30\%. NLP did not have any bearing on clinical decision-making. The Standards for QUality Improvement Reporting Excellence reporting guidelines were followed. ${ }^{16}$

\section{Statistical analysis}

Analysis was performed using Prism V.9.2 and Python V.3.7+ packages. ${ }^{17} 18$ All continuous parameters were 
Table 1 Demographic and descriptive data

\begin{tabular}{|c|c|c|c|}
\hline Variable & Group A direct to endoscopy $(n=112)$ & Group B direct to clinic $(n=111)$ & $P$ value \\
\hline Age, years, median (range) & $33.5(28-43)$ & $37(29-46)$ & 0.2076 \\
\hline Female, n (\%) & $61(54.46)$ & $60(54.05)$ & 0.9509 \\
\hline $\begin{array}{l}\text { Pre-existing diagnosis of IBD, no (\%) } \\
\text { IBD }\end{array}$ & $19(16.9)$ & $21(18.9)$ & 0.8378 \\
\hline Crohn's disease & $1(5.3)$ & $12(57.1)$ & \\
\hline Ulcerative colitis & $16(84.2)$ & $9(42.9)$ & \\
\hline IBD unclassified & $2(10.5)$ & $0(0.0)$ & \\
\hline Fecal calprotectin, median (range) & $296.5(77.5-1415.25)$ & $144.5(21.75-809.25)$ & 0.0500 \\
\hline CRP, median (range) & $4(2-12.5)$ & $3(1-10)$ & 0.2801 \\
\hline HBI, median (range) & $5(2-18)$ & $7(1-12)$ & 0.0616 \\
\hline MS, median (range) & $7(4-9.5)$ & $5.5(4-7)$ & 0.9579 \\
\hline \multicolumn{4}{|l|}{ Montreal classification } \\
\hline \multicolumn{4}{|l|}{$\begin{array}{l}\text { Crohn's disease (pre-existing and new } \\
\text { diagnosis) }\end{array}$} \\
\hline A1 - 16 years or younger & 2 & 3 & \\
\hline A2 - $17-40$ years & 16 & 22 & \\
\hline A3 - over 40 years & 3 & 6 & \\
\hline L1 - ileal & 11 & 18 & \\
\hline L2 - colonic & 4 & 6 & \\
\hline L3 - ileocolonic & 6 & 7 & \\
\hline B1 - non-stricturing, non-penetrating & 18 & 21 & \\
\hline B2 - stricturing & 2 & 10 & \\
\hline B3 - penetrating & 0 & 0 & \\
\hline$p$ - perianal & 1 & 0 & \\
\hline \multicolumn{4}{|l|}{$\begin{array}{l}\text { Ulcerative colitis/IBD-U (pre-existing and } \\
\text { new diagnosis) }\end{array}$} \\
\hline E1 - proctitis & 17 & 9 & \\
\hline E2 - left sided & 27 & 7 & \\
\hline E3 - pancolitis & 11 & 6 & \\
\hline SO - clinical remission & 4 & 2 & \\
\hline S1 - mild & 11 & 7 & \\
\hline S2 - moderate & 28 & 11 & \\
\hline S3 - severe & 12 & 2 & \\
\hline \multicolumn{4}{|l|}{ Missing data } \\
\hline FCP & 24 & 36 & \\
\hline CRP & 31 & 27 & \\
\hline
\end{tabular}

CRP, C-reactive protein ; FCP, fecal calprotectin; HBI, Harvey-Bradshow Index; IBD, inflammatory bowel disease ; IBD-U, IBD-Unclassified ; MS, Mayo Score.

summarised using either mean or median and IQR. The Mann-Whitney $U$ test compared medians, and mean differences and corresponding CIs were calculated with the Hodges-Lehmann estimate. Groups were compared using $\chi^{2}$ tests or Fisher's exact test for equality of proportions, as appropriate based on group size. Variables with a $\mathrm{p}$ value below 0.05 were considered significant.

\section{RESULTS}

We identified 247 eligible patients: 124 patients in the direct to endoscopy group (Group A) and 123 patients in the control group (Group B). See (figure 1). Twelve patients in each group did not attend their appointment $(9.67 \%$ vs $9.76 \%)$.

\section{Referral letter comparison}

Semantic analysis of all 247 referral letters ranked the four most common presenting symptoms as abdominal pain $91.09 \%(\mathrm{n}=225 / 247)$, diarrhoea $81.38 \%$ $(n=201 / 247)$, rectal bleeding 34.01\% $(n=84 / 247)$ and weight loss $32.39 \%(n=80 / 247)$.

NLP analysis revealed no significant difference between the cohorts in terms of presenting symptoms/ signs $(p=0.05119)$ and was confirmed by the manual validation exercise $(p=0.5751)$ (online supplemental table 1).

\section{Descriptive statistics}

Demographic and descriptive data such as pre-existing IBD, biomarkers and disease activity scores were 


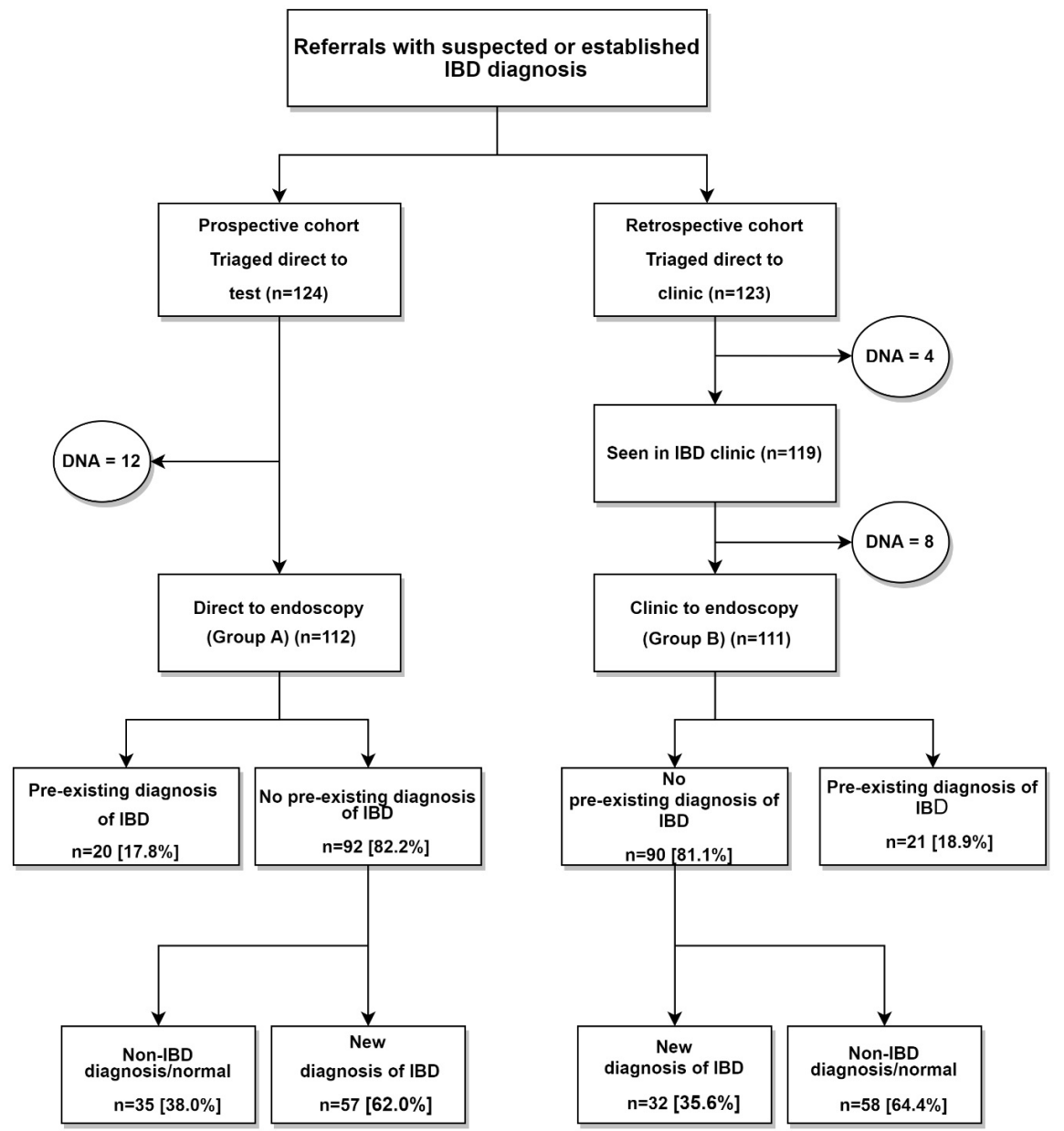

Figure 1 Breakdown of Group A and Group B participants. IBD,inflammatory bowel disease.

similar in Groups A and B; for details, (table 1). The distribution of age of onset and disease location for all patients with IBD was similar for Crohn's disease (CD), but stricturing phenotype was more common in Group B. Ulcerative colitis (UC) was more frequent, extensive and severe in Group A than Group B.

\section{Time to diagnosis}

After the introduction of the direct-access IBD endoscopy pathway, RTT fell from 177 days in (Group B) $(95 \% \mathrm{CI}$ : 157.5 to 196.5$)$ to 24 days (Group A) (95\% CI: 21.4 to 26.6 ) (difference 153 days, $\mathrm{p}<0.0001$ ), (figure 2A). This represents an $86.4 \%$
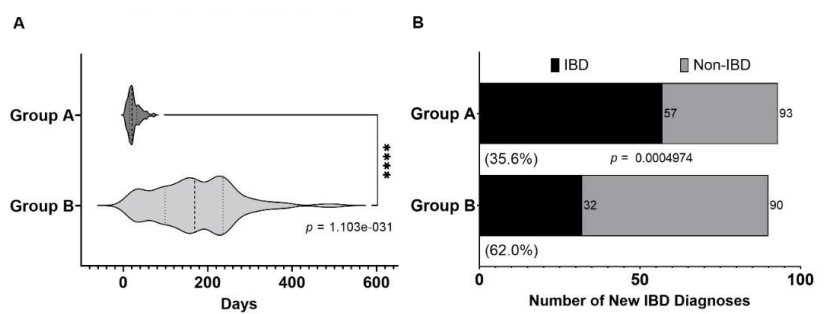

Figure 2 (A) demonstrates time to diagnosis and treatment; (B) demonstrates the diagnostic yield differences between the two cohorts. IBD, inflammatory bowel disease.
(21.9 weeks) decrease in time to clinical diagnosis, exclusion and treatment of IBD.

\section{Diagnostic yield of IBD}

Clinical diagnostic yield without pre-existing IBD was $61.2 \%(\mathrm{n}=57 / 93)$ in Group A versus $35.6 \%$ $(\mathrm{n}=32 / 90)$ in Group B, $\mathrm{p}=0.0003$, (figure 2B) and $57.9 \%(n=33 / 57)$ of new patients diagnosed with IBD in Group A had UC compared with 32.3\% $(n=10 / 31)$ in Group B. CD was less prevalent in Group A, 35.1\% $(n=20 / 57)$ compared with $61.3 \%(n=19 / 31)$ in Group B. IBD-Unclassified) was equally distributed, $7 \%$ $(n=4 / 57)$ in Group A versus 6.5\% $(n=2 / 31)$ in Group B. All patients with pre-existing IBD (Group A, $n=20$; Group $B, n=21$ ) had their diagnoses confirmed. For non-IBD outcomes (online supplemental figure 3).

\section{Type of endoscopy}

Despite a higher clinical diagnostic yield for IBD, significantly fewer colonoscopies, $46.4 \%(n=52 / 112)$, were performed in Group A compared with Group B $89.2 \%(n=99 / 111)$, OR 9.02, $\mathrm{p}<0.0001$, (figure $3 \mathrm{~A}$ ). There were no endoscopy-related complications in either group. 
A

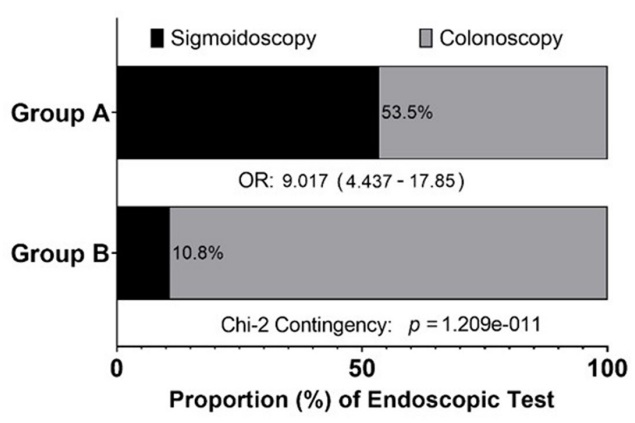

B

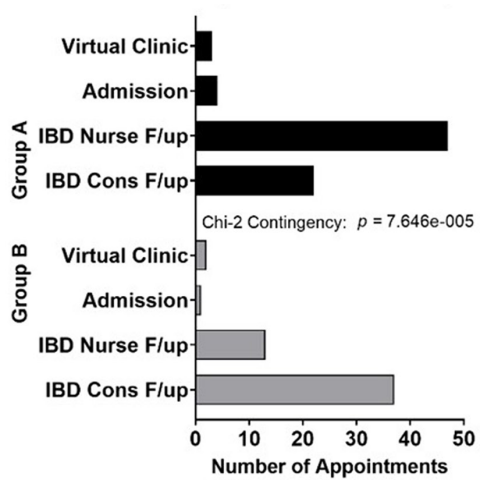

Figure 3 (A) shows the type of endoscopy requested in Group A and B, (B) shows the type of clinic follow-up between the two groups. IBD, inflammatory bowel disease.

\section{Treatment initiated}

Treatment was initiated or changed for $89.5 \%$ $(\mathrm{n}=68 / 76)$ of patients with IBD in Group A compared with $80.8 \%(n=42 / 52)$ in Group B, revealing no significant treatment differences between the groups, $\mathrm{p}=0.3051$ (online supplemental table 2). In summary, in Group A, steroids, biologics and immunomodulators were more commonly used in UC, with only immunomodulator use being more frequent for patients with CD in Group B.

\section{Follow-up}

In Group A, 61.8\% $(n=47 / 76)$ of patients referred directly to IBD physician-delivered endoscopy were followed-up in nurse-led clinics compared with $24.5 \%(n=13 / 53)$ in Group B, $\mathrm{p}<0.0001$. In contrast, $69.81 \%(n=37 / 53)$ of patients were followed-up in a consultant clinic (Group B) compared with 28.5\% $(n=22 / 76)$ in Group A, $p<0.0001$, (figure 3b). Admissions and virtual clinic follow-up were similar in both groups.

A total of 55.6\% $(n=20 / 36)$ in Group A who did not have IBD were discharged to primary care compared with 40.7\% $(n=25 / 59)$ in Group B and 25\% $(n=9 / 36)$ of patients in Group A without IBD were discharged to community dietician follow-up compared with 25.4\% $(n=14 / 59)$ in Group B (community $(n=9) /$ hospital $(\mathrm{n}=5)$ ) (online supplemental figure 3). In total, 16.7\% $(n=6 / 36)$ in Group A had consultant follow-up versus $33.9 \%(n=20 / 59)$ in Group B.

Further investigations requested between the two groups in patients who were not diagnosed with IBD are summarised in online supplemental table 3 .

\section{Patient feedback}

Patient feedback collected within this new pathway was overwhelmingly positive. Twenty-nine patients have so far completed the endoscopy pathway survey with 25 stating they felt the service provided was 'very good' and four stating they felt it was 'good' (online supplemental figure 4).

\section{DISCUSSION}

To our knowledge, this is the first real-world study to assess the clinical effectiveness of a novel direct to test IBD physician-delivered endoscopy pathway for primary care referrals with suspected IBD.

By combining assessment and treatment in our novel pathway, we have enhanced the IBD standard remit of seeing an IBD specialist within 4 weeks. We have demonstrated that it is possible to substantially reduce RTT (86\% reduction) while saving 100\% of first outpatient appointments. We achieved this by combining traditional outpatient contact with an expert endoscopic assessment leading to IBD treatment initiation in $89.5 \%$ of suspected and pre-existing IBD cases. Overall, greater use of biologics and steroids in Group $\mathrm{A}$ is likely due to a higher observed rate of UC. The absolute yield of clinical IBD diagnosis at endoscopic assessment increased from 35.6\% (Group B) to $62.0 \%$ (Group A) following the new direct-to-test pathway introduction primarily due to a higher pick-up rate of UC.

Having retrospectively compared the referral narratives with NLP, no significant difference was observed between the four-cardinal common IBD symptoms: abdominal pain, diarrhoea, rectal bleeding and weight loss. As these symptoms are similar to those for suspected colorectal cancer, patients with UC may have been diverted to a faster 2-week wait colorectal cancer pathway in Group B because of the comparatively long RTT at that time. An additional potential explanation for the difference in diagnostic yield between Group A and B might be the coronavirus pandemic. Given most data for Group A was collected during the pandemic, patients may have presented with more severe symptoms overcoming any covidrelated healthcare hesitancy.

Fecal immunofluorescence testing to screen for colorectal cancer is an established primary-care initiated low-contact endoscopy access system using a biomarker with a national referral proforma. It has a combined diagnostic yield of $7.6 \%$ for colorectal 
cancer and high-risk adenoma. ${ }^{19}$ Our diagnostic yield of IBD, by comparison, was much higher at $62.0 \%$. The difference in diagnostic yield may be due to referral letters in our pathway being assessed by experienced gastroenterology consultants triaging patients. Our locally developed electronic grading system facilitates rapid decision-making by presenting all relevant primary/secondary care information in one system. This allows for the careful selection of suitable patients for direct-access IBD endoscopy.

Interestingly, the improvement seen following the introduction of the direct-access IBD physiciandelivered endoscopy pathway was achieved using significantly fewer resources (five times greater use of flexible sigmoidoscopy in Group A) while saving all first outpatient appointments. We were also able to shift follow-up demand towards nurse-led clinics as the IBD team made treatment and management decisions at the point of endoscopy. We have demonstrated a similar effect previously for IBD flare calls directly sent to IBD physician-delivered endoscopy, freeing up outpatient capacity. ${ }^{8}$ Further additional benefits include significantly reduced IBD outpatient waiting times, up-skilling of IBD nurses, IBD-focussed gastroenterology training, the introduction of subspecialty specific IBD lists and optimisation of the IBD service in the post-covid recovery era.

Limitations of this study include that we were unable to quantify the proportion of patients referred via other channels such as the 2-week wait lower gastrointestinal pathway or referrals to colorectal surgery. The size of the cohorts was generally small in this single centre real-world setting. A further potential drawback of our IBD pathway is that dedicated IBD physicians may not be available to deliver specialised endoscopy lists at other centres. In addition, as the focus of the pathway was clinical IBD treatment decision-making at the time of endoscopy, histological confirmation was not considered. However, waiting for histological confirmation can delay treatment and does not reflect real-world clinical practice. While we have not conducted a formal health economic evaluation, we believe our pathway may prove cost-effective compared with other approaches.

Retrospective NLP (not used in clinical decisionmaking) has identified four cardinal IBD symptoms from referral letters. NLP textual analysis could simplify the administrative process for screening referral letters in the future. However, clinical decisionmaking remains with the gastroenterologist.

\section{CONCLUSION}

Our innovative direct-access IBD-physician delivered endoscopy pathway supported by our electronic grading tool is now fully integrated into clinical practice at our institution. This has transformed IBD outpatient services, saving all first outpatient appointments for suitable patients with suspected IBD. Combining the outpatient contact with the endoscopic assessment by a dedicated IBD specialist facilitates timely treatment/further management initiation within the IBD standards framework. Consideration should be given to adopting this innovative model in other suitable NHS trusts.

\section{Author affiliations}

${ }^{1}$ Department of Gastroenterology, University Hospital Southampton NHS Foundation Trust, Southampton, UK

${ }^{2}$ Clinical Informatics Research Unit, University of Southampton, Southampton, UK

${ }^{3}$ Research and Development, University Hospital Southampton NHS Foundation

Trust, Southampton, UK

${ }^{4}$ UHS Digital, University Hospital Southampton NHS Foundation Trust, Southampton, UK

${ }^{5}$ School of Medicine, University of Southampton, Southampton, UK

\section{Twitter Sohail Rahmany @gastrosprsohail}

Contributors MS data-transformation/statistics/manuscript authorship. SR design/manuscript authorship. MS/SR joint-first authorship. $\mathrm{LD} / \mathrm{FB} / \mathrm{CH} / \mathrm{RH} / \mathrm{MM} / \mathrm{SS}$ data-collection/manuscript revision. NC/BS/TRS/FC/RF manuscript revision/pathway delivery. MG design/data-analysis/manuscript authorship/ guarantor.

Funding The authors have not declared a specific grant for this research from any funding agency in the public, commercial or not-for-profit sectors.

Competing interests None declared.

Patient consent for publication Not applicable.

Ethics approval This study does not involve human participants.

Provenance and peer review Not commissioned; externally peer reviewed.

Data availability statement Data are available upon reasonable request. Available if meets ethical/research governance criteria.

Supplemental material This content has been supplied by the author(s). It has not been vetted by BMJ Publishing Group Limited (BMJ) and may not have been peer-reviewed. Any opinions or recommendations discussed are solely those of the author(s) and are not endorsed by BMJ. BMJ disclaims all liability and responsibility arising from any reliance placed on the content. Where the content includes any translated material, BMJ does not warrant the accuracy and reliability of the translations (including but not limited to local regulations, clinical guidelines, terminology, drug names and drug dosages), and is not responsible for any error and/or omissions arising from translation and adaptation or otherwise.

Open access This is an open access article distributed in accordance with the Creative Commons Attribution Non Commercial (CC BY-NC 4.0) license, which permits others to distribute, remix, adapt, build upon this work noncommercially, and license their derivative works on different terms, provided the original work is properly cited, appropriate credit is given, any changes made indicated, and the use is noncommercial. See: http://creativecommons.org/licenses/by-nc/4. $0 /$.

\section{ORCID iDs}

Matthew Stammers http://orcid.org/0000-0003-3850-3116

Sohail Rahmany http://orcid.org/0000-0003-0924-2690

Trevor R Smith http://orcid.org/0000-0003-1614-2946

Fraser Cummings http://orcid.org/0000-0002-9659-3247

\section{REFERENCES}

1 Ibd benchmarking tool, 2019. Available: https://ibduk.org/ibdbenchmarking-tool [Accessed 11 Oct 2021].

2 The Hidden Cost and a Vision for Change Crohn's and Colitis Care in the UK, 2021. Available: https://s3.eu-west-2. 
amazonaws.com/files.ibduk.org/documents/CROJ8096IBD-National-Report-WEB-210427-2.pdf? mtime $=$ 20210616102531\&focal=none [Accessed 10 Sep 2021].

3 Blackwell J, Saxena S, Jayasooriya N, et al. Prevalence and duration of gastrointestinal symptoms before diagnosis of inflammatory bowel disease and predictors of timely specialist review: a population-based study. J Crohns Colitis 2021;15:203-11.

4 Elective Care Transformation NHS England. Transforming gastroenterology elective care services a Handbook for local health and care systems, 2017. https://www.england.nhs.uk/ wp-content/uploads/2017/11/gastroenterology-elective-carehandbook.pdf

5 Magro F, Gionchetti P, Eliakim R, et al. Third European evidence-based consensus on diagnosis and management of ulcerative colitis. Part 1: definitions, diagnosis, extraintestinal manifestations, pregnancy, cancer surveillance, surgery, and ileo-anal pouch disorders. J Crohns Colitis 2017;11:649-70.

6 Gomollón F, Dignass A, Annese V, et al. 3rd European evidence-based consensus on the diagnosis and management of Crohn's disease 2016: Part 1: diagnosis and medical management. J Crohns Colitis 2017;11:3-25.

7 Magro F, Langner C, Driessen A, et al. European consensus on the histopathology of inflammatory bowel disease. J Crohns Colitis 2013;7:827-51.

8 Harris R, McDonnell M, Downey ML. OTH-03 impact of direct-access IBD physician delivered sigmoidoscopy from acute IBD flare line call service. Gastroenterology Service 2019:A222.1-A222.

9 Sarkar S, Livingstone R, Borca F. Development of a novel electronic referral grading \& triage system. Accept Poster Present BSG Conf, 2021.
10 Jackson R, Kartoglu I, Stringer C, et al. CogStack - experiences of deploying integrated information retrieval and extraction services in a large National Health Service Foundation Trust hospital. BMC Med Inform Decis Mak 2018;18:47.

11 Nehme F, Feldman K. Evolving role and future directions of natural language processing in gastroenterology. Dig Dis Sci 2021;66:29-40.

12 Chen D, Fulmer C, Gordon IO, et al. Application of Artificial Intelligence to Clinical Practice in Inflammatory Bowel Disease - What the Clinician Needs to Know. J Crohns Colitis 2021:jjab169.

13 NHS England. NHS England " Referral to treatment, 2015. Available: https://www.england.nhs.uk/rtt/ [Accessed 10 Sep 2021].

14 Kraljevic Z, Bean D, Mascio A. MedCAT - Medical Concept Annotation Tool. ArXiv191210166 Cs Stat 2019 http://arxiv. org/abs/1912.10166

15 Mohan S, Li D. MedMentions: a large biomedical corpus annotated with UMLS concepts. ArXiv190209476 Cs 2019 http://arxiv.org/abs/1902.09476

16 Ogrinc G, Davies L, Goodman D, et al. SQUIRE 2.0 (Standards for QUality Improvement Reporting Excellence) : revised publication guidelines from a detailed consensus process: Table 1. BMJ Qual Saf 2016;25:986-92.

17 GraphPad prism version 9.3 for widowns GraphPad software. Available: https://www.graphpad.com/scientific-software/prism/

18 Python Software Foundation. Python language reference, version 3.7. Available: http://www.python.org

19 D'Souza N, Georgiou Delisle T, Chen M, et al. Faecal immunochemical test is superior to symptoms in predicting pathology in patients with suspected colorectal cancer symptoms referred on a $2 \mathrm{WW}$ pathway: a diagnostic accuracy study. Gut 2021;70:1130-8. 